\title{
Supplementary materials Adaptive empirical Bayesian smoothing splines
}

\author{
Paulo Serra $^{*, \ddagger}$ and Tatyana Krivobokova ${ }^{\dagger}$
}

\section{Technical results and proofs}

\subsection{Demmler-Reinsch basis and estimating equations}

Let $\left\{\psi_{i}\right\}_{i=1}^{\infty}$ denote the Demmler-Reinsch basis of $\mathcal{W}_{\beta}(M)$, such that it holds

$$
\int_{0}^{1} \psi_{\beta, i}(x) \psi_{\beta, j}(x) d x=\delta_{i j}=\nu_{\beta, i}^{-1} \int_{0}^{1} \psi_{\beta, i}^{(\beta)}(x) \psi_{\beta, j}^{(\beta)}(x) d x
$$

Hence, any $f \in \mathcal{W}_{\beta}(M)$ can be represented as $f=\sum_{i=1}^{\infty} \theta_{\beta, i} \psi_{\beta, i}$ for $\theta_{\beta, i}=\int_{0}^{1} f(x) \psi_{\beta, i}(x) d x$, and $\left\|f^{(\beta)}\right\|^{2}=\sum_{i=1}^{\infty} \theta_{\beta, i}^{2} \nu_{\beta, i}<M^{2}$. We also define Sobolev spaces for real-valued $\beta$ s via

$$
\mathcal{W}_{\beta}(M)=\left\{f: f \in \mathcal{C}^{\lfloor\beta\rfloor-1}[0,1],\left\|f^{(\beta)}\right\|^{2}=\sum_{i=1}^{\infty} \theta_{\beta, i}^{2} \nu_{\beta, i}^{\beta /\lfloor\beta\rfloor}<M^{2}\right\}
$$

The Demmler-Reinsch basis of the natural spline space of degree $2 q-1$ with knots at observations $\mathcal{S}_{2 q-1}(\boldsymbol{x})$ is defined via

$$
\sum_{k=1}^{n} \phi_{q, i}\left(x_{k}\right) \phi_{q, j}\left(x_{k}\right)=\delta_{i j}=\eta_{q, i}^{-1} \int_{0}^{1} \phi_{q, i}^{(q)}(x) \phi_{q, j}^{(q)}(x) d x .
$$

With the Demmler-Reinsch basis the smoother matrix can be represented as $\boldsymbol{S}_{\lambda, q}=$ $\boldsymbol{\Phi}_{q}\left\{\boldsymbol{I}_{n}+\lambda n \operatorname{diag}\left(\boldsymbol{\eta}_{q}\right)\right\}^{-1} \boldsymbol{\Phi}_{q}^{T}$, where $\boldsymbol{\Phi}_{q}=\boldsymbol{\Phi}_{q}(\boldsymbol{x})=\left[\phi_{q, 1}(\boldsymbol{x}), \ldots, \phi_{q, n}(\boldsymbol{x})\right]=\left[\phi_{q, j}\left(x_{i}\right)\right]_{i, j=1}^{n}$. For the eigenvalues $\boldsymbol{\eta}_{q}$, Theorem 2.2. in Speckman (1985) establishes the following approximation

$$
\eta_{q, 1}=0, \ldots, \eta_{q, q}=0, \quad n \eta_{q, i}=\pi^{2 q}(i-q)^{2 q}\{1+o(1)\}, i=q+1, \ldots, n,
$$

where the $o(1)$ term is uniform over $i=o\left\{n^{2 /(2 q+1)}\right\}$, as $n \rightarrow \infty$.

For $q=\beta$ in Speckman (1985) is shown that $\sqrt{n} \phi_{\beta, i}=\psi_{\beta, i}\{1+o(1)\}, n \eta_{\beta, i}=$ $\nu_{\beta, i}\{1+o(1)\}, n^{-1} B_{\beta, i}^{2}=\theta_{\beta, i}^{2}\{1+o(1)\}$, as well as $n^{-1} \sum_{i=\beta+1}^{n} B_{\beta, i}^{2} n \eta_{\beta, i}=\left\|f^{(\beta)}\right\|^{2}\{1+$

\footnotetext{
* Korteweg-de Vries Institute for Mathematics, University of Amsterdam, Amsterdam, the Netherlands, p.serra@uva.nl.

$\dagger$ Institute for Mathematical Stochastics, Georg-August-Universität Göttingen, Göttingen, Germany, tkrivob@gwdg.de.

$\ddagger$ The research took place while the author was a postdoctoral researcher at Institute for Mathematical Stochastics, Georg-August-Universität Göttingen, Göttingen, Germany.
}

(C) 0000 International Society for Bayesian Analysis

DOI: 0000 
$o(1)\}$, where $B_{\beta, i}=\sum_{j=1}^{n} \phi_{\beta, j}\left(x_{i}\right) f\left(x_{i}\right)$. In case $\beta \neq q$, that is $f \in \mathcal{W}_{\beta}(M)$, but the Demmler-Reinsch basis of degree $q$ is used, we find in a similar fashion

$$
\frac{1}{n} \sum_{i=q+1}^{n} B_{q, i}^{2}\left(n \eta_{q, i}\right)^{\min (\beta, q) / q}=\left\|f^{(\min (\beta, q))}\right\|^{2}\{1+o(1)\} .
$$

The estimating equation $T_{\lambda}(\lambda, q)$ defined in Section 2 is derived in the same way as those in Krivobokova (2013) using the fact that $d \boldsymbol{S}_{\lambda, q} / d \lambda=-\lambda^{-1}\left(\boldsymbol{S}_{\lambda, q}-\boldsymbol{S}_{\lambda, q}^{2}\right) ; T_{q}(\lambda, q)$ follows in the same way with a few extra remarks. Firstly, to determine the derivatives of eigenvalues $\eta_{q, i}$ with respect to $q$ we employ representation (31). Secondly, we use that $\sum_{i=1}^{n} X_{q, i}^{2}=\boldsymbol{Y}^{t} \boldsymbol{\Phi}_{q} \boldsymbol{\Phi}_{q}^{t} \boldsymbol{Y}=\sum_{i=1}^{n} Y_{i}^{2}$, which is independent of $q$ and implies that the contribution of $\partial X_{i}^{2} / \partial q$ is negligible. This can be seen as follows. For $j=j(n) \propto \lambda^{-\alpha /(2 q)}$ with any $\alpha \in(0,1)$, so that $\lambda n \eta_{j}=o(1)$, similar to the proof of Lemma 2 in Krivobokova (2013) follows

$$
\begin{aligned}
\frac{1}{n} \sum_{i=1}^{n} \frac{\partial X_{q, i}^{2}}{\partial q} \frac{\lambda n \eta_{q, i}}{1+\lambda n \eta_{q, i}} & =\frac{1}{n} \sum_{i=1}^{n} \frac{\partial X_{q, i}^{2}}{\partial q}-\frac{1}{n} \sum_{i=1}^{n} \frac{\partial X_{q, i}^{2}}{\partial q} \frac{1}{1+\lambda n \eta_{q, i}} \\
& =\frac{1}{n} \sum_{i=j+1}^{n} \frac{\partial X_{q, i}^{2}}{\partial q} \frac{\lambda n \eta_{q, i}}{1+\lambda n \eta_{q, i}}\{1+o(1)\},
\end{aligned}
$$

that is, $\partial X_{q, i}^{2} / \partial q$ contributes only to the tail part of the sum. Next, observe that $X_{q, i} / \sqrt{n}=B_{q, i} / \sqrt{n}+O_{p}(\sigma / \sqrt{n})$, so that

$$
\frac{1}{n} \sum_{i=j+1}^{n} \frac{\partial X_{q, i}^{2}}{\partial q} \frac{\lambda n \eta_{q, i}}{1+\lambda n \eta_{q, i}}=\frac{2 \sigma}{\sqrt{n}} \sum_{i=j+1}^{n} \frac{\partial B_{q, i} / \sqrt{n}}{\partial q}\left\{O_{p}(1)+B_{q, i} / \sigma\right\} \frac{\lambda n \eta_{q, i}}{1+\lambda n \eta_{q, i}} .
$$

Since $n^{-1} \sum_{i=1}^{n} B_{q, i}^{2} n \eta_{q, i}<\infty$, we can bound $\left|B_{q, i}\right| / \sqrt{n} \leq \operatorname{const}(i-q)^{-(2 q+1+\epsilon) / 2}$ for some $\epsilon>0$ and for $i>j$ it follows that $B_{q, i}=O(1)$ for $\lambda=O\left(n^{-2 q /\{\alpha(2 q+1+\epsilon)\}}\right)$, which includes $\lambda_{q}$ for a suitable choice of $\alpha \in(0,1)$. Since $\left(\lambda n \eta_{q, i}\right)^{\delta} /\left(1+\lambda n \eta_{q, i}\right) \leq 1$, for some $\delta \in[0,1]$, showing that $\left|\partial B_{q, i} / \partial q\right|=O(1)\left|B_{q, i}\right|$ will imply that

$$
\begin{aligned}
\left|\frac{1}{n} \sum_{i=j+1}^{n} \frac{\partial X_{q, i}^{2}}{\partial q} \frac{\lambda n \eta_{q, i}}{1+\lambda n \eta_{q, i}}\right| & \leq \frac{2 \sigma}{\sqrt{n}} \sum_{i=j+1}^{n}\left|\frac{B_{q, i}}{\sqrt{n}}\right|\left(\lambda n \eta_{q, i}\right)^{\min (\beta, q) / 2 q} O_{p}(1) \\
& =\frac{\lambda^{\min (\beta, q) / q}}{\sqrt{\lambda^{\min (\beta, q) / q} n}} o_{p}(1) .
\end{aligned}
$$

To see that $\left|\partial B_{q, i} / \partial q\right|=O(1)\left|B_{q, i}\right|$, note that $\sum_{i=1}^{n} B_{i}^{2}=\sum_{i=1}^{n} f\left(x_{i}\right)^{2}$ is independent of $q$. Moreover, $B_{i}^{2} / n$ is a non-increasing function of $q$ for all $i>q$, so that $\partial\left(\sum_{i=1}^{q} B_{i}^{2}\right) / \partial q=-\partial\left(\sum_{i=q+1}^{n} B_{i}^{2}\right) / \partial q$. Since $B_{i}=\sum_{j=1}^{n} x_{j}^{i-1} f\left(x_{j}\right)$ for $i \leq q$ and $\sum_{i=1}^{q} x^{i-1}=\left(x^{q}-1\right) /(x-1)$, we get

$$
\frac{\partial}{\partial q} \sum_{i=1}^{q} B_{i}^{2}=\frac{\partial}{\partial q} \sum_{j, k} f\left(x_{j}\right) f\left(x_{k}\right) \frac{\left(x_{j} x_{k}\right)^{q}-1}{\left(x_{j} x_{k}\right)-1}=\sum_{j, k} f\left(x_{j}\right) f\left(x_{k}\right) \frac{\left(x_{j} x_{k}\right)^{q} \log \left(x_{j} x_{k}\right)}{\left(x_{j} x_{k}\right)-1}
$$




$$
=\sum_{j, k} f\left(x_{j}\right) f\left(x_{k}\right) \frac{\left(x_{j} x_{k}\right)^{q}-1}{\left(x_{j} x_{k}\right)-1} \frac{\left(x_{j} x_{k}\right)^{q} \log \left(x_{j} x_{k}\right)}{\left(x_{j} x_{k}\right)^{q}-1}=O(1) \sum_{i=1}^{q} B_{i}^{2} .
$$

In a similar fashion and using that $\sum_{i=q+1}^{n} \phi_{i}\left(x_{j}\right) \phi_{i}\left(x_{j}\right)=\mathbb{I}(j=k)-\sum_{i=1}^{q}\left(x_{j} x_{k}\right)^{i-1}$, follows

$$
\frac{\partial}{\partial q} \sum_{i=q+1}^{n} B_{i}^{2}=O(1) \sum_{i=q+1}^{n} B_{i}^{2}
$$

Since $B_{i}^{2}$ for $i>q$ is a function of eigenvalues, which are proportional to $(i-q)^{2 q}$, changing summation in the left hand side sum and interchanging summation and differentiation imply the result.

We conclude that the contribution of $\partial X_{q, i}^{2} / \partial q$ is negligible and therefore subindices $q$ in $X_{q, i}$ and $B_{q, i}$ will be subsequently omitted.

\subsection{Auxiliary lemmas}

Lemma 1. Let $\boldsymbol{S}_{\lambda, q}$ be the smoother matrix. Then, for all $q>1 / 2, l \in \mathbb{N}, m \in \mathbb{N} \cup\{0\}$, and $\lambda$ such that $\lambda \rightarrow 0, n \lambda \rightarrow \infty$, as $n \rightarrow \infty$,

$$
\operatorname{tr}\left\{\left(\boldsymbol{I}_{n}-\boldsymbol{S}_{\lambda, q}\right)^{m} \boldsymbol{S}_{\lambda, q}^{l}\right\}=\sum_{i=1}^{n} \frac{\left(\lambda n \eta_{q, i}\right)^{m}}{\left(1+\lambda n \eta_{q, i}\right)^{m+l}}=\lambda^{-1 /(2 q)} \kappa_{q}(m, l)\{1+o(1)\}
$$

where the $o(1)$ term depends only on $m$ and $l$, and the constants $\kappa_{q}(m, l)$ are

$$
\kappa_{q}(m, l)=\frac{\Gamma\{m+1 /(2 q)\} \Gamma\{l-1 /(2 q)\}}{2 \pi q \Gamma(l+m)} .
$$

Proof. See Krivobokova (2013).

Lemma 2. Consider $\boldsymbol{\eta}_{q, i}$ as in (31). Then, as $n \rightarrow \infty$ such that $\lambda \rightarrow 0$ for all $m \in$ $\mathbb{N} \cup\{0\}, r, l \in \mathbb{N}, q>1 / 2$

$$
\sum_{i=q+1}^{n} \frac{\left(\lambda n \eta_{q, i}\right)^{m}\left\{\log \left(n \eta_{q, i}\right)\right\}^{r}}{\left(1+\lambda n \eta_{q, i}\right)^{m+l}}=\lambda^{-1 /(2 q)}\{\log (1 / \lambda)\}^{r} \kappa_{q}(m, l)\{1+o(1)\} .
$$

Proof. Follows the same lines as Lemma 1.

Lemma 3. Let $m \in \mathbb{N}, q>1 / 2$, and $\lambda \rightarrow 0$ such that $\lambda n \rightarrow \infty$. If $f \in \mathcal{W}_{q}$,

$$
\frac{1}{n} \sum_{i=q+1}^{n} \frac{B_{i}^{2} \lambda n \eta_{q, i} \log \left(\lambda n \eta_{q, i}\right)}{\left(1+\lambda n \eta_{q, i}\right)^{m}}=-\lambda \log (1 / \lambda)\left\|f^{(q)}\right\|^{2}\{1+o(1)\} .
$$


If $f \in \mathcal{W}_{\beta} \cap \mathcal{M}$, but $f \notin \mathcal{W}_{\beta+\epsilon}, \epsilon>0$, and $q>\beta>1 / 2$, then

$$
\begin{gathered}
\frac{1}{n} \sum_{i=q+1}^{n} \frac{B_{i}^{2} \lambda n \eta_{q, i} \log \left(\lambda n \eta_{q, i}\right)}{\left(1+\lambda n \eta_{q, i}\right)^{m}} \geq c \lambda^{\beta / q}, \\
\frac{1}{n} \sum_{i=q+1}^{n} \frac{B_{i}^{2} \lambda n \eta_{q, i}}{\left(1+\lambda n \eta_{q, i}\right)^{m}} \geq c \lambda^{\beta / q}
\end{gathered}
$$

for some sufficiently small constant $c>0$. If $f$ is a polynomial of degree $d-1$ (such that $f \in \mathcal{P}_{d}$ and $f \notin \mathcal{P}_{d-1}$ ) and $q \geq d$ then

$$
\frac{1}{n} \sum_{i=q+1}^{n} \frac{B_{i}^{2} \lambda n \eta_{q, i} \log \left(\lambda n \eta_{q, i}\right)}{\left(1+\lambda n \eta_{q, i}\right)^{m}}=0
$$

Proof. We remind that if $f \in \mathcal{W}_{q}$ then

$$
\frac{1}{n} \sum_{i=q+1}^{n} B_{i}^{2} n \eta_{q, i}=\left\|f^{(q)}\right\|^{2}\{1+o(1)\}<\infty .
$$

Obviously, if $f$ is a polynomial of degree $d-1, d \in \mathbb{N}\left(f \in \mathcal{P}_{d}, f \notin \mathcal{P}_{d-1}\right)$, then $\left\|f^{(q)}\right\|=0, q \geq d$ and (36) follows.

To prove (33) let $j=j_{n}(\alpha)$ be like in the proof of Lemma 2 in Krivobokova (2013); more specifically, take $j=\pi^{-1} \lambda^{-\alpha /(2 q)}$ for $\alpha \in(0,1)$, so that $\lambda n \eta_{q, j}=o(1)$; following the proof of that lemma, for each $\alpha \in(0,1)$,

$\frac{1}{n} \sum_{i=q+1}^{n} \frac{B_{i}^{2} \lambda n \eta_{q, i} \log \left(\lambda n \eta_{q, i}\right)}{\left(1+\lambda n \eta_{q, i}\right)^{m}}=-\frac{\lambda}{n}\left[\sum_{i=q+1}^{j} B_{i}^{2} n \eta_{q, i} \log \frac{1}{\lambda n \eta_{q, i}}\{1+o(1)\}-O\left(\sum_{i=j+1}^{n} B_{i}^{2} n \eta_{q, i}\right)\right]$.

If $f \in \mathcal{W}_{q}$ such that $n^{-1} \sum_{i=q+1}^{n} B_{i}^{2} n \eta_{q, i}=\left\|f^{(q)}\right\|^{2}\{1+o(1)\}<\infty$, whence for any choice of $j \rightarrow \infty$ the second term on the right-hand-side is negligible compared to the first. Conclude that for each $\alpha \in(0,1)$,

$\lambda \log \lambda\left\|f^{(q)}\right\|^{2}\{1+o(1)\} \leq \frac{1}{n} \sum_{i=q+1}^{n} \frac{B_{i}^{2} \lambda n \eta_{q, i} \log \left(\lambda n \eta_{q, i}\right)}{\left(1+\lambda n \eta_{q, i}\right)^{m}} \leq(1-\alpha) \lambda \log \lambda\left\|f^{(q)}\right\|^{2}\{1+o(1)\}$,

(for an $o(1)$ term which is uniform over $\alpha$ ) such that (33) follows by taking $\alpha \rightarrow 0$.

Finally, we establish (34). Let $f \in \mathcal{W}_{\beta}(M), f \notin \mathcal{W}_{\beta+\epsilon}, \epsilon>0, q>\beta+\epsilon$. Consider the function

$$
g_{r}(x)=\frac{x^{r} \log (x)}{(1+x)^{m}}, \quad r \in(0,1), x>0 .
$$

This continuous function is negative on the interval $(0,1)$ and positive on $(1, \infty)$. It converges to zero when $x \rightarrow 0$, has a global minimum at some point $m_{r} \in(0,1)$, is 
zero at $x=1$, has a global maximum at $M_{r} \in(1, \infty)$, and converges monotonically to zero as $x \rightarrow \infty$. Let $j_{-2} \leq j_{-1} \leq j_{0} \leq j_{1} \leq j_{2}$ be sequences of integers depending on $n$ such that: $g_{1-\beta / q}\left(\lambda n \eta_{q_{j_{-2}}}\right)=o(1)$ and $g_{1-\beta / q}\left(\lambda n \eta_{q, j_{-1}}\right)=o(1)$ with $\lambda n \eta_{q, j_{-2}}<$ $m_{1-\beta / q}<\lambda n \eta_{q, j_{-1}}$ and $j_{-2} \rightarrow \infty ; \lambda n \eta_{q, j_{0}}<1 \leq \lambda n \eta_{q, j_{0}+1}$; and for some (small) constant $0<c<g_{1}\left(M_{1}\right), g_{1}\left(\lambda n \eta_{q, i}\right) \geq c, i=j_{1}, \ldots, j_{2}$, such that $g_{1}\left(\lambda n \eta_{q, j_{1}-1}\right)<c$, $g_{1}\left(\lambda n \eta_{q, j_{2}+1}\right)<c$. (Note that $m_{r}, M_{r}$, and the sequences $j_{k}, k=-2 \ldots, 2$ depend on $r$ and $m$.)

Splitting the summation along these sequences, and using the bounds above

$$
\begin{aligned}
& \frac{1}{n} \sum_{i=q+1}^{j_{0}} \frac{B_{i}^{2} \lambda n \eta_{q, i} \log \left(\lambda n \eta_{q, i}\right)}{\left(1+\lambda n \eta_{q, i}\right)^{m}} \geq \\
& \quad \geq-\lambda^{\beta / q} \frac{1}{n}\left\{o(1) \sum_{i \in I} B_{i}^{2}\left(n \eta_{q, i}\right)^{\beta / q}-g_{1-\beta / q}\left(m_{1-\beta / q}\right) \sum_{i=j_{-2}+1}^{j_{-1}} B_{i}^{2}\left(\lambda n \eta_{q, i}\right)^{\beta / q}\right\},
\end{aligned}
$$

where $I=\left\{q+1, \ldots, j_{-2}\right\} \cup\left\{j_{-1}, \ldots, j_{0}\right\}$. Since by definition $f \in \mathcal{W}_{\beta}$, then we have $n^{-1} \sum_{i=q+1}^{n} B_{i}^{2}\left(\lambda n \eta_{q, i}\right)^{\beta / q}=\left\|f^{(\beta)}\right\|^{2}\{1+o(1)\}<\infty$, so, since $j_{-2} \rightarrow \infty$ we conclude that the lower bound in the previous display is $-o\left(\lambda^{\beta / q}\right)\left\|f^{(\beta)}\right\|^{2}$, which lower bounds the sum of the negative terms. As for the sum of the positive terms,

$$
\frac{1}{n} \sum_{i=j_{0}+1}^{n} \frac{B_{i}^{2} \lambda n \eta_{q, i} \log \left(\lambda n \eta_{q, i}\right)}{\left(1+\lambda n \eta_{q, i}\right)^{m}} \geq \frac{1}{n} \sum_{i=j_{1}}^{j_{2}} \frac{B_{i}^{2} \lambda n \eta_{q, i} \log \left(\lambda n \eta_{q, i}\right)}{\left(1+\lambda n \eta_{q, i}\right)^{m}} \geq \frac{c}{n} \sum_{i=j_{1}}^{j_{2}} B_{i}^{2} .
$$

Simple computations show we can take $O\left(\lambda^{-1 /(2 q)}\right)=j_{1} \leq j_{2} / \rho$, for any $\rho>2$. By the polished-tail condition the previous display can further be lower bounded by

$$
\frac{c}{n} \sum_{i=j_{1}}^{\rho j_{1}} B_{i}^{2} \geq \frac{c\left(n \eta_{q, \rho j_{1}}\right)^{-\frac{\beta+\epsilon}{q}}}{n} \sum_{i=j_{1}}^{\rho j_{1}} B_{i}^{2}\left(n \eta_{q, i}\right)^{\frac{\beta+\epsilon}{q}} \geq \frac{O\left(j_{1}^{-2(\beta+\epsilon)}\right)}{n L} \sum_{i=j_{1}}^{n} B_{i}^{2}\left(n \eta_{q, i}\right)^{\frac{\beta+\epsilon}{q}} .
$$

The sum $\sum_{i=j_{1}}^{n} B_{i}^{2}\left(n \eta_{q, i}\right)^{\frac{\beta+\epsilon}{q}} / n$ converges to infinity as long as $j_{1}=o(n), \epsilon>0$. The power $j_{1}^{-2(\beta+\epsilon)}$ is $o\left(\lambda^{\beta / q}\right)$ for every $\epsilon>0$ but it is $O\left(\lambda^{\beta / q}\right)$ if $\epsilon=0$. Conclude that for small enough $\epsilon$, namely such that $j_{1}^{-2(\beta+\epsilon)}$ is of a larger order than the $o\left(\lambda^{\beta / q}\right)$ term from the bound on the negative terms, the sum of the positive terms dominates. In fact, picking an appropriate sequence $\epsilon \rightarrow 0$ one can show that the sum of the positive terms is lower bounded by a small enough multiple of $\lambda^{\beta / q}$. The bound in (34) follows. The bound in (35) follows in the same way by adjusting the definition of the sequences $j_{1}$ and $j_{2}$.

Lemma 4 (Lemma 3 from Krivobokova, 2013). Let $\lambda \rightarrow 0$ be such that $n \lambda \rightarrow \infty$. Then, for any $l \in \mathbb{N}$,

$$
\begin{aligned}
\left.\boldsymbol{f}^{T}\left(\boldsymbol{I}_{n}-\boldsymbol{S}_{\lambda, q}\right) \boldsymbol{S}_{\lambda, q}^{1+l} \boldsymbol{f}\right|_{\lambda=\lambda_{q}} & =\left.\sigma^{2} \operatorname{tr}\left(\boldsymbol{S}_{\lambda, q}^{2}\right)\right|_{\lambda=\lambda_{q}}\{1+o(1)\}, \\
\left.\boldsymbol{f}^{T}\left(\boldsymbol{I}_{n}-\boldsymbol{S}_{\lambda, q}\right)^{1+l} \boldsymbol{S}_{\lambda, q} \boldsymbol{f}\right|_{\lambda=\lambda_{q}} & =o\left(\lambda_{q}^{-1 /(2 q)}\right) .
\end{aligned}
$$


Lemma 5. Let $T_{C_{p}}(\lambda)$ be the estimating equation for $\lambda_{f}$ as defined in Krivobokova (2013), $\lambda_{f}$ the solution to $\mathbb{E} T_{C_{p}}(\lambda)=0$, and $\hat{\lambda}_{f}$ the solution to $T_{C_{p}}(\lambda)=0$. Then

$$
\mathbb{E}\left\{\lambda_{f}^{-1 /(4 q)}\left(\hat{\lambda}_{f} / \lambda_{f}-1\right)\right\}^{2} \leq \frac{4 \kappa_{q}(4,2)}{\left\{4 \kappa_{q}(1,2)-3 \kappa_{q}(1,3)\right\}^{2}}\{1+o(1)\},
$$

such that in particular $\mathbb{V}\left(\hat{\lambda}_{f} / \lambda_{f}-1\right)=o(1)$.

Proof. By definition of $\hat{\lambda}_{f}$, for some $\tilde{\lambda}$ between $\hat{\lambda}_{f}$ and $\lambda_{f}$,

$$
0=T_{C_{p}}\left(\hat{\lambda}_{f}\right)=T_{C_{p}}\left(\lambda_{f}\right)+T_{C_{p}}^{\prime}(\tilde{\lambda})\left(\hat{\lambda}_{f}-\lambda_{f}\right) .
$$

It is known (cf. the supplementary materials to Krivobokova, 2013) that,

$$
\begin{aligned}
\mathbb{V}\left\{T_{C_{p}}\left(\lambda_{f}\right)\right\} & =2 \frac{\sigma^{4}}{n^{2}} \kappa_{q}(4,2) \lambda_{f}^{-1 /(2 q)}\{1+o(1)\}, \\
\mathbb{E}\left\{T_{C_{p}}^{\prime}\left(\tau \lambda_{f}\right)\right\} & =\mathbb{E}\left\{T_{C_{p}}^{\prime}\left(\lambda_{f}\right)\right\}\left\{1+\frac{2 q-1}{2 q} O(\tau-1)\right\} \\
& =\frac{\sigma^{2}}{n} \lambda_{f}^{-1-1 /(2 q)}\left(4 \kappa_{q}(1,2)-3 \kappa_{q}(1,3)\right)\left\{1+\frac{2 q-1}{2 q} O(\tau-1)\right\},
\end{aligned}
$$

for $\tau \in[1-\epsilon, 1+\epsilon]$ and small enough $\epsilon>0$. Let $A(\epsilon)$ be the event $\left\{\left|\hat{\lambda}_{f} / \lambda_{f}-1\right|<\epsilon\right\}$, and write $\tilde{\lambda}=\tau \lambda_{f}$, so that

$$
\begin{aligned}
2 \frac{\sigma^{4}}{n^{2}} & \kappa_{q}(4,2) \lambda_{f}^{-1 /(2 q)}\{1+o(1)\}= \\
& =\mathbb{E}\left\{\left(\hat{\lambda}_{f}-\lambda_{f}\right)^{2} \mathbb{E}\left[T_{C_{p}}^{\prime}(\tilde{\lambda})^{2} \mid \hat{\lambda}_{f}\right]\right\} \geq \mathbb{E}\left\{\left(\hat{\lambda}_{f}-\lambda_{f}\right)^{2} \mathbb{E}\left[T_{C_{p}}^{\prime}\left(\tau \lambda_{f}\right)^{2} \mid \hat{\lambda}_{f}, A(\epsilon)\right] \mathbb{P}(A(\epsilon))\right\} \\
& =\mathbb{E}\left\{\left(\hat{\lambda}_{f}-\lambda_{f}\right)^{2} \mathbb{E}\left[T_{C_{p}}^{\prime}\left(\tau \lambda_{f}\right) \mid A(\epsilon)\right]^{2}\{1+o(1)\}\right\} \\
& =\mathbb{E}\left\{\left(\hat{\lambda}_{f}-\lambda_{f}\right)^{2} \mathbb{E}\left[T_{C_{p}}^{\prime}\left(\lambda_{f}\right)\right]^{2} \mathbb{E}\left[1+\frac{2 q-1}{2 q} O(\tau-1) \mid A(\epsilon)\right]^{2}\{1+o(1)\}\right\},
\end{aligned}
$$

where we use the fact that by definition $\mathbb{E}\left\{T_{C_{p}}\left(\lambda_{f}\right)\right\}=0$, and Jensen's inequality. Since the previous display holds for all small enough $\epsilon$, set $\epsilon$ so that $1+(2 q-1) O(\tau-1) /(2 q) \geq$ $1 / \sqrt{2}$, say, whence

$$
\mathbb{E}\left\{\hat{\lambda}_{f} / \lambda_{f}-1\right\}^{2} \leq \lambda_{f}^{1 /(2 q)} \frac{4 \kappa_{q}(4,2)}{\left\{4 \kappa_{q}(1,2)-3 \kappa_{q}(1,3)\right\}^{2}}\{1+o(1)\}=o(1) .
$$

\subsection{Proof of Theorem 2}

Assume $f \in L_{2}$. First, note that for some $\tilde{\lambda}$ between $\hat{\lambda}_{q}$ and $\lambda_{q}$, a.s.,

$$
T_{q}\left(\hat{\lambda}_{q}, q\right)=T_{q}\left(\lambda_{q}, q\right)+\left.\left(\hat{\lambda}_{q}-\lambda_{q}\right) \frac{\partial T_{q}(\lambda, q)}{\partial \lambda}\right|_{\lambda=\tilde{\lambda}} .
$$


We have

$$
\begin{aligned}
\mathbb{V} T_{q}\left(\lambda_{q}, q\right) & =\frac{2 \sigma^{2}}{q^{2} n^{2}}\left[\frac{\left\{\log \left(1 / \lambda_{q}\right)\right\}^{2}}{\lambda_{q}^{1 /(2 q)} / \kappa_{q}(2,2)} \sigma^{2}\{1+o(1)\}+2 \sum_{i=q+1}^{n} B_{i}^{2}\left\{\frac{\lambda_{q} n \eta_{q, i} \log \left(n \eta_{q, i}\right)}{\left(1+\lambda_{q} n \eta_{q, i}\right)^{2}}\right\}^{2}\right] \\
& =O\left\{\lambda_{q}^{-1 /(2 q)} \log \left(1 / \lambda_{q}\right)^{2} n^{-2}\right\} \rightarrow 0, \quad n \rightarrow \infty .
\end{aligned}
$$

Hence, $T_{q}\left(\lambda_{q}, q\right)-\mathbb{E} T_{q}\left(\lambda_{q}, q\right) \stackrel{P}{\longrightarrow} 0$ for any $q>1 / 2$. In fact, for any $\epsilon>0$, and $\mathbb{Q}_{n}$ as defined at the end of Section 2,

$$
\begin{aligned}
& \mathbb{P}\left(\max _{q \in \mathbb{Q}_{n}}\left|T_{q}\left(\lambda_{q}, q\right)-\mathbb{E} T_{q}\left(\lambda_{q}, q\right)\right|>\epsilon\right) \leq \mathbb{P}\left(\sum_{q \in \mathbb{Q}_{n}}\left|T_{q}\left(\lambda_{q}, q\right)-\mathbb{E} T_{q}\left(\lambda_{q}, q\right)\right|>\epsilon\right) \leq \\
& \sum_{q \in \mathbb{Q}_{n}} \mathbb{P}\left(\left|T_{q}\left(\lambda_{q}, q\right)-\mathbb{E} T_{q}\left(\lambda_{q}, q\right)\right|>\epsilon /\left|\mathbb{Q}_{n}\right|\right) \leq \frac{\left|\mathbb{Q}_{n}\right|^{3} w}{\epsilon^{2}} \max _{q \in \mathbb{Q}_{n}} \mathbb{V} T_{q}\left(\lambda_{q}, q\right),
\end{aligned}
$$

by Chebyshev's inequality. We assume without loss of generality that $\left|\mathbb{Q}_{n}\right|=O\left\{1 / \log (n)^{\alpha}\right\}$, for some $\alpha>2$ such that the upper-bound in the previous display converges to zero, implying that $T_{q}\left(\lambda_{q}, q\right)-\mathbb{E} T_{q}\left(\lambda_{q}, q\right) \stackrel{P}{\longrightarrow} 0$ takes place uniformly over $q \in \mathbb{Q}_{n}$. Note also that $\mathbb{V} T_{q}\left(\lambda_{q}, q\right)=o\left[\left\{\mathbb{E} T_{q}\left(\lambda_{q}, q\right)\right\}^{2}\right]$. The remaining term in (37) is controlled using the same arguments as Lemma 5 so we omit the details.

Consider a polished tail $f \in \mathcal{L}_{2}$. Without loss of generality say that $T_{q}, q \notin \mathbb{Q}_{n}$, is obtained by linear interpolation of the values of $T_{q}$ at $\mathbb{Q}_{n}$. From the discussion in Sections 3.1 and 3.2 , the criterion $\mathbb{E} T_{q}\left(\lambda_{q}, q\right)$ is strictly negative for $q \leq \bar{\beta}$ and is positive for $q>\bar{\beta}$, whence

$$
\begin{aligned}
& \mathbb{P}\left\{\max _{q \in\left\{r \in \mathbb{Q}_{n}: r<\bar{\beta}\right\}} T_{q}\left(\hat{\lambda}_{q}, q\right)<0, \min _{q \in\left\{r \in \mathbb{Q}_{n}: r>\bar{\beta}\right\}} T_{q}\left(\hat{\lambda}_{q}, q\right)>0\right\} \leq \\
& \quad \leq \mathbb{P}\left\{\sup _{q \in(1 / 2, \bar{\beta})} T_{q}\left(\hat{\lambda}_{q}, q\right)<0, \inf _{q \in(\bar{\beta}, \log (n)]} T_{q}\left(\hat{\lambda}_{q}, q\right)>0\right\} \leq \mathbb{P}\{\hat{q}=\bar{\beta}\},
\end{aligned}
$$

The first inequality follows from the fact that we linearly interpolate the criterion, and the second inequality follows from the definition of $\hat{q}$. From the computations above, the left-hand-side of the previous display converges to 1 as $n \rightarrow \infty$, so that the second statement of the theorem follows. For $f \in \mathcal{W}_{\beta}(M), \beta>1 / 2$, by the same arguments as above,

$$
\begin{aligned}
& \mathbb{P}\left[\max _{q \in\left\{r \in \mathbb{Q}_{n}: r<\beta\right\}} T_{q}\left(\hat{\lambda}_{q}, q\right)<0, \min _{q \in\left\{r \in \mathbb{Q}_{n}: r>\beta\right\}} T_{q}\left(\hat{\lambda}_{q}, q\right)=o_{p}\left\{T_{q}\left(\hat{\lambda}_{\beta}, \beta\right)\right\}\right] \leq \\
& \quad \leq \mathbb{P}\left[\sup _{q \in(1 / 2, \beta)} T_{q}\left(\hat{\lambda}_{q}, q\right)<0, \inf _{q \in(\beta, \log (n)]} T_{q}\left(\hat{\lambda}_{q}, q\right)=o_{p}\left\{T_{q}\left(\hat{\lambda}_{\beta}, \beta\right)\right\}\right] \leq \mathbb{P}[\beta \leq \hat{q} \leq \log (n)],
\end{aligned}
$$

and the first statement of the theorem follows since the left-hand-side of the previous display converges to 1 , as $n \rightarrow \infty$. The last statement of the theorem follows by using the exact same argument. 


\subsection{Proof of Theorem 3}

If we assume that for some countable set $Q_{n} \in \mathbb{Q}_{n}$,

$$
\inf _{\boldsymbol{f} \in \mathcal{W}} \mathbb{P}_{f}\left(\hat{q} \in Q_{n}\right)=1+o(1),
$$

then (25) follows if we show that uniformly over $q \in Q_{n}$,

$$
\inf _{\boldsymbol{f} \in \mathcal{W}} \mathbb{P}_{f}\left\{\left\|\boldsymbol{f}-\hat{\boldsymbol{f}}_{\hat{\lambda}_{q}, q}\right\| \leq \hat{\sigma}_{\hat{\lambda}_{q}, q} L r_{n}\left(\hat{\lambda}_{q}, q\right)\right\}=1+o\left(1 /\left|Q_{n}\right|\right)
$$

For each $q \in Q_{n}, f \in \mathcal{W}$, consider intervals $L_{q}=\left[\underline{\lambda}_{q}, \bar{\lambda}_{q}\right]$ - in fact $L_{q}(f)=\left[\underline{\lambda}_{q}(f), \bar{\lambda}_{q}(f)\right]$ - with $\lambda_{q} \in L_{q}$ and $\bar{\lambda}_{q} / \underline{\lambda}_{q} \rightarrow 1$, such that

$$
\inf _{\boldsymbol{f} \in \mathcal{W}} \mathbb{P}_{f}\left\{\hat{\lambda}_{q} \in L_{q}(f)\right\}=1+o(1) .
$$

If there exist constants $C_{1}, C_{2}, C_{3}, C_{4}$, such that uniformly over $f \in \mathcal{W}$ and $q \in Q_{n}$,

$$
\begin{aligned}
& \inf _{\lambda \in L_{q}} r_{n}^{2}(\lambda, q) \geq C_{1}^{2} \frac{\bar{\lambda}_{q}^{-1 /(2 q)}}{n}, \\
& \inf _{f \in \mathcal{W}} \mathbb{P}_{f}\left(\inf _{\lambda \in L_{q}} \hat{\sigma}_{\lambda, q}^{2} \geq C_{2}^{2}\right)=1+o(1), \\
& \sup _{\lambda \in L_{q}}\left\|\mathbb{E}_{f} \hat{\boldsymbol{f}}_{\lambda, q}-\boldsymbol{f}\right\|^{2} \leq C_{3}^{2} \frac{\bar{\lambda}_{q}^{-1 /(2 q)}}{n} \\
& \inf _{f \in \mathcal{W}} \mathbb{P}_{f}\left(\sup _{\lambda \in L_{q}}\left\|\hat{\boldsymbol{f}}_{\lambda, q}-\mathbb{E}_{f} \hat{\boldsymbol{f}}_{\lambda, q}\right\|^{2} \leq C_{4}^{2} \bar{\lambda}_{q}^{-1 /(2 q)} / n\right)=o\left(1 /\left|Q_{n}\right|\right),
\end{aligned}
$$

then, if $L$ is large enough such that $C_{4}+C_{3} \leq L C_{2} C_{1}$, conclude

$\inf _{f \in \mathcal{W}} \mathbb{P}_{f}\left\{\sup _{\lambda \in L_{q}}\left\|\hat{\boldsymbol{f}}_{\lambda, q}-\mathbb{E}_{f} \hat{\boldsymbol{f}}_{\lambda, q}\right\|+\sup _{\lambda \in L_{q}}\left\|\mathbb{E}_{f} \hat{\boldsymbol{f}}_{\lambda, q}-\boldsymbol{f}\right\| \leq \inf _{\lambda \in L_{q}} \hat{\sigma}_{\lambda, q} L r_{n}(\lambda, q)\right\}=1+o\left(1 /\left|Q_{n}\right|\right)$,

such that by the triangle inequality, (39) holds.

If (38) holds, then (26) follows if we show that uniformly over $q \in Q_{n}$,

$$
\inf _{f \in \mathcal{W}_{\beta}(M)} \mathbb{P}_{f}\left\{r_{n}\left(\hat{\lambda}_{q}, q\right) \leq K n^{-\beta /(2 \beta+1)}\right\}=1+o\left(1 /\left|Q_{n}\right|\right)
$$

Since the risk of the smoothing spline satisfies (4), it is only possible to establish (45) if $q \geq \beta$. Consider $s_{n}(\beta)=\max \left\{q \in \mathbb{Q}_{n}: q \leq \beta\right\}$. By the first and second statements of Theorem 2 we conclude that if we take $Q_{n}=\mathbb{Q}_{n} \cap\left(s_{n}, \bar{\beta}+1\right)$, then $\mathbb{P}\left(\hat{q} \in Q_{n}\right)=1+o(1)$, such that every $q \in Q_{n}$ is such that $q \geq \beta$. Note also that $\mathbb{Q}_{n}$ can be taken such that $\left|Q_{n}\right|=o\left\{\log (n)^{2}\right\}$, say. These facts are used when checking (42), (44), and (45) below.

Condition (40) follows from the consistency results in Section 3 so that it suffices to check conditions (41)-(45), which we do in the remainder of this section. 


\section{Checking condition (41)}

The sequence $r_{n}(\lambda, q)$ is implicitly defined by

$\mathbb{P}\left\{R_{n}(\lambda, q) \leq r_{n}(\lambda, q)^{2}\right\}=1-\alpha, \quad$ with $\quad R_{n}(\lambda, q) \sim \frac{1}{N} \boldsymbol{Z}^{T} \boldsymbol{S}_{\lambda, q} \boldsymbol{Z} \sim \frac{1}{N} \sum_{i=q+1}^{n} \frac{\epsilon_{i}^{2}}{1+\lambda n \eta_{q, i}}$,

where $\boldsymbol{Z} \sim N\left(\mathbf{0}, \boldsymbol{I}_{n}\right)$ and $N \sim \mathcal{X}_{n}^{2}$, with $\boldsymbol{Z}$ and $N$ independent, and $\boldsymbol{\epsilon}=\boldsymbol{\Phi}^{T} \boldsymbol{Z} \sim$ $N\left(\mathbf{0}, \boldsymbol{I}_{n}\right)$. Since $R_{n}(\lambda, q)$ decreases with $\lambda$, the infimum in (41) is attained at $\lambda=\bar{\lambda}_{q}$. Set $R=R_{n}\left(\bar{\lambda}_{q}, q\right), r=r_{n}\left(\bar{\lambda}_{q}, q\right)$ and note that by the one-sided Chebychev's inequality, $r^{2} \geq \mathbb{E} R-\{(1-\alpha) / \alpha\}^{1 / 2}(\mathbb{V} R)^{1 / 2}$. Further, since $N^{-1}$ is independent of $\boldsymbol{\epsilon}$, and has an inverse-chi-squared distribution with $n$ degrees of freedom such that $\mathbb{E} N^{-1}=(n-2)^{-1}$,

$$
\mathbb{E} R=\mathbb{E} \frac{1}{N} \sum_{i=q+1}^{n} \frac{\mathbb{E} \epsilon_{i}^{2}}{1+\bar{\lambda}_{q} n \eta_{q, i}}=\frac{1}{n-2} \operatorname{tr}\left(\boldsymbol{S}_{\bar{\lambda}_{q}, q}\right)=\frac{\bar{\lambda}_{q}^{-1 /(2 q)}}{n-2} \kappa_{q}(0,1)\{1+o(1)\} .
$$

By similar computations, since $\mathbb{V} N^{-1}=2(n-2)^{-2}(n-4)^{-1}$,

$$
\mathbb{V} R=3\left(\mathbb{E} \frac{1}{N}\right)^{2} \operatorname{tr}\left(\boldsymbol{S}_{\bar{\lambda}_{q}, q}^{2}\right)+\mathbb{V} \frac{1}{N} \operatorname{tr}\left(\boldsymbol{S}_{\bar{\lambda}_{q}, q}\right)^{2}+3 \mathbb{V} \frac{1}{N} \operatorname{tr}\left(\boldsymbol{S}_{\bar{\lambda}_{q}, q}^{2}\right)=o\left\{(\mathbb{E} R)^{2}\right\} .
$$

Conclude that (41) holds for $C_{1}^{2}<\kappa_{q}(0,1)\{1+o(1)\}$.

\section{Checking condition (42)}

We may take $L_{q}=\left[c \bar{\lambda}_{q}, \bar{\lambda}_{q} / c\right]$, for some $c=1+o(1)<1$. For $\lambda \in L_{q}$, we bound, a.s.,

$$
\hat{\sigma}_{\lambda, q}^{2}=\frac{1}{n} \boldsymbol{Y}^{T}\left(\boldsymbol{I}_{n}-\boldsymbol{S}_{\lambda, q}\right) \boldsymbol{Y} \geq \frac{c^{2}}{n} \sum_{i=q+1}^{n} \frac{X_{i}^{2} \bar{\lambda}_{q} n \eta_{q, i}}{1+\bar{\lambda}_{q} n \eta_{q, i}} \geq \frac{c^{2}}{n} \boldsymbol{Y}^{T}\left(\boldsymbol{I}_{n}-\boldsymbol{S}_{\bar{\lambda}_{q}, q}\right) \boldsymbol{Y}
$$

Denote this lower-bound by $S=S_{n}\left(\bar{\lambda}_{q}, q, c\right)$. By Chebyshev's inequality,

$$
\mathbb{P}\left(\inf _{\lambda \in L_{q}} \hat{\sigma}_{\lambda, q}^{2} \geq C_{2}^{2}\right) \geq 1-\mathbb{P}\left\{S \leq \mathbb{E} S-\left(\mathbb{E} S-C_{2}^{2}\right)\right\} \geq 1-\frac{\mathbb{V} S}{\mathbb{V} S+\left(\mathbb{E} S-C_{2}^{2}\right)^{2}} .
$$

Using Lemma 1 we have

$\mathbb{E} S=\frac{c^{2}}{n}\left\{\sigma^{2} \operatorname{tr}\left(\boldsymbol{I}_{n}-\boldsymbol{S}_{\bar{\lambda}_{q}, q}\right)+\boldsymbol{f}^{T}\left(\boldsymbol{I}_{n}-\boldsymbol{S}_{\bar{\lambda}_{q}, q}\right) \boldsymbol{f}\right\} \geq c^{2} \sigma^{2}-c^{2} \sigma^{2} \frac{\operatorname{tr}\left(\boldsymbol{S}_{\bar{\lambda}_{q}, q}\right)}{n}=\sigma^{2}\{1+o(1)\}$.

By Lemma 1, and the bound on $\boldsymbol{f}^{T}\left(\boldsymbol{I}_{n}-\boldsymbol{S}_{\bar{\lambda}_{q}, q}\right)^{2} \boldsymbol{f}$ from the next section,

$$
\mathbb{V} S=\frac{1}{n^{2}}\left[2 \sigma^{4} \operatorname{tr}\left\{\left(\boldsymbol{I}_{n}-\boldsymbol{S}_{\bar{\lambda}_{q}, q}\right)^{2}\right\}+4 \sigma^{2} \boldsymbol{f}^{T}\left(\boldsymbol{I}_{n}-\boldsymbol{S}_{\bar{\lambda}_{q}, q}\right)^{2} \boldsymbol{f}\right]=O\left(\frac{\mathbb{E} S}{n}\right) .
$$

Condition (42) holds by taking $C_{2}^{2}<\sigma^{2}\{1+o(1)\}$. Since the bound in previous display is $o\left\{1 / \log (n)^{2}\right\}$ we further assume that the bound holds uniformly over $q \in Q_{n}$. 


\section{Checking condition (43)}

Let $L_{q}$ be as in the previous section. For $\lambda \in L_{q}$ the squared bias term satisfies

$$
\left\|\mathbb{E} \hat{\boldsymbol{f}}_{\lambda, q}-\boldsymbol{f}\right\|^{2}=\frac{1}{n} \sum_{i=q+1}^{n} \frac{B_{i}^{2}\left(\lambda n \eta_{q, i}\right)^{2}}{\left(1+\lambda n \eta_{q, i}\right)^{2}} \leq \frac{1}{n} \sum_{i=q+1}^{n} \frac{B_{i}^{2}\left(\bar{\lambda}_{q} n \eta_{q, i}\right)^{2}}{\left(1+\bar{\lambda}_{q} n \eta_{q, i}\right)^{2}} \leq \frac{1}{c^{2}}\left\|\left(\boldsymbol{S}_{\lambda_{q}, q}-\boldsymbol{I}_{n}\right) \boldsymbol{f}\right\|^{2} .
$$

By Lemma 4 and simple manipulations,

$$
\left\|\left(\boldsymbol{S}_{\lambda_{q}, q}-\boldsymbol{I}_{n}\right) \boldsymbol{f}\right\|^{2}=\frac{1}{n} \boldsymbol{f}^{T}\left(\boldsymbol{S}_{\lambda_{q}, q}-\boldsymbol{I}_{n}\right) \boldsymbol{S}_{\lambda_{q}, q} \boldsymbol{f}-\frac{1}{n} \boldsymbol{f}^{T}\left(\boldsymbol{S}_{\lambda_{q}, q}-\boldsymbol{I}_{n}\right) \boldsymbol{f} \leq \sigma^{2} \frac{\operatorname{tr}\left(\boldsymbol{S}_{\lambda_{q}, q}\right)}{n}\{1+o(1)\} .
$$

The statement follows by Lemma 1 , for $C_{3}^{2}>\sigma^{2} \kappa_{q}(0,1)\{1+o(1)\}$.

\section{Checking condition (44)}

For $L_{q}$ as in the previous sections, uniformly over $\lambda \in L_{q}$ the variance term satisfies, a.s.,

$$
\left\|\hat{\boldsymbol{f}}_{\lambda, q}-\mathbb{E} \hat{\boldsymbol{f}}_{\lambda, q}\right\|^{2}=\left\|\boldsymbol{S}_{\lambda, q}(\boldsymbol{Y}-\boldsymbol{f})\right\|^{2}=\frac{1}{n} \sum_{i=q+1}^{n} \frac{\left(X_{i}-B_{i}\right)^{2}}{\left(1+\lambda n \eta_{q, i}\right)^{2}} \leq \frac{1}{n} \sum_{i=q+1}^{n} \frac{\left(X_{i}-B_{i}\right)^{2}}{\left(1+\underline{\lambda}_{q} n \eta_{q, i}\right)^{2}},
$$

where $\boldsymbol{X}-\boldsymbol{B}=\boldsymbol{\Phi}^{T}(\boldsymbol{Y}-\boldsymbol{f}) \sim N\left(\mathbf{0}, \sigma^{2} \boldsymbol{I}_{n}\right)$. Denote the upper-bound in the previous display by $V=V_{n}\left(\underline{\lambda}_{q}, q\right)$. By Lemma 1 , the expectation of $V$ is

$\mathbb{E} V=\frac{\sigma^{2}}{n} \operatorname{tr}\left(\boldsymbol{S}_{\underline{\lambda}_{q}, q}^{2}\right)=\frac{\sigma^{2}}{n} \underline{\lambda}_{q}^{-1 /(2 q)} \kappa_{q}(0,2)\{1+o(1)\}=\sigma^{2} c^{-1 / q} \kappa_{q}(0,2) \frac{\bar{\lambda}_{q}^{-1 /(2 q)}}{n}\{1+o(1)\} ;$

the respective variance is $\mathbb{V} V=3 \sigma^{2} n^{-2} \operatorname{tr}\left(\boldsymbol{S}_{\underline{\underline{\lambda}}_{q}, q}^{4}\right)=O(\mathbb{E} V / n)$. Condition (44) follows by an application of the one-sided Chebyshev's inequality for $C_{4}^{2}>\sigma^{2} \kappa_{q}(0,2)\{1+o(1)\}$. Since the bound in previous display is $o\left\{1 / \log (n)^{2}\right\}$ we further assume that the bound holds uniformly over $q \in Q_{n}$ with an $o(1)$ term that is $o\left(1 /\left|Q_{n}\right|\right)$.

\section{Checking condition (45)}

Note that if (40) holds then it suffices to check

$$
\inf _{f \in \mathcal{W}_{\beta}(M)} \mathbb{P}_{f}\left\{\sup _{\lambda \in L_{q}} r_{n}(\lambda, q) \leq K n^{-\beta /(2 \beta+1)}\right\}=\inf _{f \in \mathcal{W}_{\beta}(M)} 1_{\left\{D_{n}(f)\right\}}=1+o(1),
$$

where $D_{n}(f)=\left\{\sup _{\lambda \in L_{q}(f)} r_{n}(\lambda, q) \leq K n^{-\beta /(2 \beta+1)}\right\}$.

Proceeding as in Section 8.4, the supremum in $D_{n}(f)$ is attained at $\underline{\lambda}_{q}$ so if we redefine $R=R_{n}\left(\underline{\lambda}_{q}, q\right)$ and $r=r_{n}\left(\underline{\lambda}_{q}, q\right)$, then by the one-sided Chebyshev's inequality $r^{2} \leq \mathbb{E} R+\{\alpha /(1-\alpha)\}^{1 / 2}(\mathbb{V} R)^{1 / 2}$. By direct substitution, since $\beta \leq q<\bar{b}$, we have that for sufficiently large $n$

$$
\mathbb{E} R=\frac{\lambda_{q}^{-1 /(2 q)}}{n} \kappa_{q}(0,1)\{1+o(1)\} \leq K n^{-2 \beta /(2 \beta+1)}, \quad \text { and } \quad \mathbb{V} R=o\left\{(\mathbb{E} R)^{2}\right\}
$$


for constant $K$ depending on $\beta, \bar{b}, \sigma^{2}$, and $M$. Since the bound on the variance in previous display is $o\left\{1 / \log (n)^{2}\right\}$ we further assume that the bound holds uniformly over $q \in Q_{n}$. Note that in particular, if $q=\beta$,

$$
\left[\left\|f^{(\beta)}\right\|^{2} /\left\{\sigma^{2} \kappa_{\beta}(0,2)\right\}\right]^{1 /(2 \beta+1)} \leq\left[M^{2} /\left\{\sigma^{2} \kappa_{\beta}(0,2)\right\}\right]^{1 /(2 \beta+1)}=K,
$$

uniformly over $f \in \mathcal{W}_{\beta}$. The statement of the theorem follows.

\subsection{Proof of Theorem 4}

For simplicity, we assume in the following that $\hat{f}_{\lambda, q}$ is independent of $\hat{\lambda}_{f}$ (say we make two independent observations at each design point in $\boldsymbol{x}$; this certainly does not impair the performance of the GCV based estimate of $\lambda$, and it considerably simplifies the exposition).

Expanding around the oracle $\lambda_{f}$ we have, a.s.,

$$
\frac{1}{\left(1+n \eta_{q, i} \hat{\lambda}_{f}\right)^{m}}=\frac{1}{\left(1+n \eta_{q, i} \lambda_{f}\right)^{m}}-m \frac{n \eta_{q, i} \lambda_{f}}{\left(1+n \eta_{q, i} \lambda_{f}\right)^{m+1}} O\left(\hat{\lambda}_{f} / \lambda_{f}-1\right) .
$$

Conclude that $\operatorname{tr}\left(\boldsymbol{S}_{\hat{\lambda}_{f}, q}^{m}\right)=\operatorname{tr}\left(\boldsymbol{S}_{\lambda_{f}, q}^{m}\right)-m \operatorname{tr}\left\{\left(\boldsymbol{I}_{n}-\boldsymbol{S}_{\lambda_{f}, q}\right) \boldsymbol{S}_{\lambda_{f}, q}^{m}\right\} O\left(\hat{\lambda}_{f} / \lambda_{f}-1\right)$, a.s., and that $\boldsymbol{f}^{T} \boldsymbol{S}_{\hat{\lambda}_{f}, q}^{m} \boldsymbol{f}=\boldsymbol{f}^{T} \boldsymbol{S}_{\lambda_{f}, q}^{m} \boldsymbol{f}-m \boldsymbol{f}^{T}\left(\boldsymbol{I}_{n}-\boldsymbol{S}_{\lambda_{f}, q}\right) \boldsymbol{S}_{\lambda_{f}, q}^{m} \boldsymbol{f} O\left(\hat{\lambda}_{f} / \lambda_{f}-1\right)$, a.s..

We now determine the asymptotic expectation and variance of risk of $\hat{f}_{\hat{\lambda}_{f}, q}$. From $n\left\|\hat{\boldsymbol{f}}_{\hat{\lambda}_{f}, q}-\boldsymbol{f}\right\|^{2}=\boldsymbol{Y}^{T} \boldsymbol{S}_{\hat{\lambda}_{f}, q}^{2} \boldsymbol{Y}-2 \boldsymbol{Y}^{T} \boldsymbol{S}_{\hat{\lambda}_{f}, q} \boldsymbol{f}+\boldsymbol{f}^{T} \boldsymbol{f}$, and the expansions above

$$
\begin{aligned}
\mathbb{E}\left[n\left\|\hat{\boldsymbol{f}}_{\hat{\lambda}_{f}, q}-\boldsymbol{f}\right\|^{2} \mid \hat{\lambda}_{f}\right] & =\sigma^{2} \operatorname{tr}\left(\boldsymbol{S}_{\hat{\lambda}_{f}, q}^{2}\right)+\boldsymbol{f}^{T}\left(\boldsymbol{I}_{n}-\boldsymbol{S}_{\hat{\lambda}_{f}, q}\right)^{2} \boldsymbol{f}= \\
& =\left\{\sigma^{2} \operatorname{tr}\left(\boldsymbol{S}_{\lambda_{f}, q}^{2}\right)+\boldsymbol{f}^{T}\left(\boldsymbol{I}_{n}-\boldsymbol{S}_{\lambda_{f}, q}\right)^{2} \boldsymbol{f}\right\}\left\{1+O\left(\frac{\hat{\lambda}_{f}}{\lambda_{f}}-1\right)\right\}, \\
\mathbb{V}\left[n\left\|\hat{\boldsymbol{f}}_{\hat{\lambda}_{f}, q}-\boldsymbol{f}\right\|^{2} \mid \hat{\lambda}_{f}\right] & =2 \sigma^{4} \operatorname{tr}\left(\boldsymbol{S}_{\hat{\lambda}_{f}, q}^{4}\right)+4 \sigma^{2} \boldsymbol{f}^{T}\left(\boldsymbol{I}_{n}-\boldsymbol{S}_{\hat{\lambda}_{f}, q}\right)^{2} \boldsymbol{S}_{\hat{\lambda}_{f}, q}^{2} \boldsymbol{f}= \\
& =\left\{2 \sigma^{4} \operatorname{tr}\left(\boldsymbol{S}_{\lambda_{f}, q}^{4}\right)+4 \sigma^{2} \boldsymbol{f}^{T}\left(\boldsymbol{I}_{n}-\boldsymbol{S}_{\lambda_{f}, q}\right)^{2} \boldsymbol{S}_{\lambda_{f}, q}^{2} \boldsymbol{f}\right\}\left\{1+O\left(\frac{\hat{\lambda}_{f}}{\lambda_{f}}-1\right)\right\},
\end{aligned}
$$

by Lemma 1, and by Lemma 4 . By Lemma 5 we conclude that

$$
\begin{aligned}
& \mathbb{E}\left\{\mathbb{E}\left[\left\|\hat{\boldsymbol{f}}_{\hat{\lambda}_{f}, q}-\boldsymbol{f}\right\|^{2} \mid \hat{\lambda}_{f}\right]\right\}=\frac{1}{n}\left\{\sigma^{2} \operatorname{tr}\left(\boldsymbol{S}_{\lambda_{f}, q}^{2}\right)+\boldsymbol{f}^{T}\left(\boldsymbol{I}_{n}-\boldsymbol{S}_{\lambda_{f}, q}\right)^{2} \boldsymbol{f}\right\}\{1+o(1)\}, \\
& \mathbb{V}\left\{\mathbb{E}\left[\left\|\hat{\boldsymbol{f}}_{\hat{\lambda}_{f}, q}-\boldsymbol{f}\right\|^{2} \mid \hat{\lambda}_{f}\right]\right\}=\frac{1}{n^{2}} o\left\{\sigma^{2} \operatorname{tr}\left(\boldsymbol{S}_{\lambda_{f}, q}^{2}\right)+\boldsymbol{f}^{T}\left(\boldsymbol{I}_{n}-\boldsymbol{S}_{\lambda_{f}, q}\right)^{2} \boldsymbol{f}\right\}^{2}, \\
& \mathbb{E}\left\{\mathbb{V}\left[\left\|\hat{\boldsymbol{f}}_{\hat{\lambda}_{f}, q}-\boldsymbol{f}\right\|^{2} \mid \hat{\lambda}_{f}\right]\right\}=\frac{1}{n^{2}}\left\{2 \sigma^{4} \operatorname{tr}\left(\boldsymbol{S}_{\lambda_{f}, q}^{4}\right)+4 \sigma^{2} \boldsymbol{f}^{T}\left(\boldsymbol{I}_{n}-\boldsymbol{S}_{\lambda_{f}, q}\right)^{2} \boldsymbol{S}_{\lambda_{f}, q}^{2} \boldsymbol{f}\right\}\{1+o(1)\},
\end{aligned}
$$

so that again by Lemma 1 , and by Lemma 4 ,

$$
\mathbb{V}\left(\left\|\hat{\boldsymbol{f}}_{\hat{\lambda}_{f}, q}-\boldsymbol{f}\right\|^{2}\right)=o\left\{\mathbb{E}\left(\left\|\hat{\boldsymbol{f}}_{\hat{\lambda}_{f}, q}-\boldsymbol{f}\right\|^{2}\right)\right\}^{2} .
$$


Now, if $\mathbb{E}\left(\left\|\hat{\boldsymbol{f}}_{\hat{\lambda}_{f}, q}-\boldsymbol{f}\right\|^{2}\right)>C \sigma^{2} r_{n}\left(\lambda_{\beta}, \beta\right)^{2}, C>1$, then by Chebyshev's inequality,

$$
\begin{aligned}
\mathbb{P}\left(\boldsymbol{f} \in \hat{\mathcal{D}}_{n}\right) & =\mathbb{P}\left\{\left\|\hat{\boldsymbol{f}}_{\hat{\lambda}_{f}, q}-\boldsymbol{f}\right\|^{2} \leq \sigma^{2} r_{n}\left(\lambda_{\beta}, \beta\right)^{2}\right\} \leq \\
& \leq\left[1+\left\{\mathbb{E}\left(\left\|\hat{\boldsymbol{f}}_{\hat{\lambda}_{f}, q}-\boldsymbol{f}\right\|^{2}\right)-\sigma^{2} r_{n}\left(\lambda_{\beta}, \beta\right)^{2}\right\}^{2} / \mathbb{V}\left(\left\|\hat{\boldsymbol{f}}_{\hat{\lambda}_{f}, q}-\boldsymbol{f}\right\|^{2}\right)\right]^{-1}=o(1) .
\end{aligned}
$$

From Sections 8.4, $r_{n}(\lambda, q)^{2}=\kappa_{q}(0,1) \lambda^{-1 /(2 q)} / n\{1+o(1)\}$. By the definition of $\lambda_{f}$ (cf. Krivobokova, 2013),

$$
\begin{aligned}
& \mathbb{E}\left(\left\|\hat{\boldsymbol{f}}_{\hat{\lambda}_{f}, q}-\boldsymbol{f}\right\|^{2}\right) \geq \frac{\sigma^{2}}{n} \operatorname{tr}\left(\boldsymbol{S}_{\lambda_{f}, q}^{2}\right)+\frac{1}{n} \boldsymbol{f}^{T}\left(\boldsymbol{I}_{n}-\boldsymbol{S}_{\lambda_{f}, q}\right)^{2} \boldsymbol{S}_{\lambda_{f}, q} \boldsymbol{f} \\
& \quad=\frac{\sigma^{2}}{n}\left\{2 \operatorname{tr}\left(\boldsymbol{S}_{\lambda_{f}, q}^{2}\right)-\operatorname{tr}\left(\boldsymbol{S}_{\lambda_{f}, q}^{3}\right)\right\}\{1+o(1)\}=\frac{\sigma^{2}}{n}\left\{2 \kappa_{q}(0,2)-\kappa_{q}(0,3)\right\} \lambda_{f}^{-1 /(2 q)}\{1+o(1)\} .
\end{aligned}
$$

From (17) and (27),

$$
\lambda_{\beta}^{-1 /(2 \beta)} \geq\left\{\kappa_{q}(1,2) / \kappa_{\beta}(0,2)\right\}^{1 /(2 \beta+1)} \lambda_{f}^{-1 /(2 q)}\{1+o(1)\} .
$$

By basic properties of the gamma function, for any $\beta>1 / 2$,

$$
2 \kappa_{q}(0,2)-\kappa_{q}(0,3)>\kappa_{\beta}(0,1)\left\{\kappa_{q}(1,2) / \kappa_{\beta}(0,2)\right\}^{1 /(2 \beta+1)}, \quad q \in[\beta / 2, \beta],
$$

so that the probability that $\boldsymbol{f}$ belongs to $\hat{\mathcal{D}}_{n}$ is indeed $o(1)$.

\section{References}

Krivobokova, T. (2013). "Smoothing parameter selection in two frameworks for penalized splines." J. R. Statist. Soc. B, 75: 725-741. 2, 3, 4, 5, 6, 11

Speckman, P. (1985). "Spline smoothing and optimal rates of convergence in nonparametric regression models." Ann. Statist., 13: 970-983. 1 